\title{
Improved electrical properties of metal-oxide-semiconductor capacitor with HfTiON gate dielectric by using HfSiON interlayer
}

\author{
J. P. Xu and F. Ji \\ Department of Electronic Science and Technology, Huazhong University of Science and Technology, Wuhan \\ 430074, People's Republic of China \\ C. X. Li and P. T. Lai ${ }^{\mathrm{a})}$ \\ Department of Electrical and Electronic Engineering, the University of Hong Kong, Pokfulam Road, Hong \\ Kong \\ J. G. Guan \\ State Key Laboratory of Advanced Technology for Materials Synthesis and Processing, Wuhan University of \\ Science and Technology, Wuhan 430070, People's Republic of China \\ Y. R. Liu \\ Institute of Polymer Optoelectronic Materials and Devices, South China University of Technology, \\ Guangzhou 510640, People's Republic of China and The School of Physics Science andam Technology, \\ South China University of Technology, Guangzhou 510640, People's Republic of China
}

(Received 17 August 2007; accepted 23 September 2007; published online 10 October 2007)

\begin{abstract}
Metal-oxide-semiconductor (MOS) capacitor with $\mathrm{HfTiON} / \mathrm{HfSiON}$ stack structure as high- $k$ gate dielectric is fabricated, and its electrical properties are compared with those of a similar device with HfTiON only as gate dielectric. Experimental results show that the device with HfTiON/HfSiON gate dielectric exhibits better interface properties, lower gate leakage current, and enhanced high-field reliability. All these improvements should be attributed to the fact that the HfSiON buffer layer effectively blocks the diffusion of $\mathrm{Ti}$ atoms to the $\mathrm{Si}$ substrate, thus resulting in a $\mathrm{SiO}_{2} / \mathrm{Si}$-like HfSiON/Si interface. (c) 2007 American Institute of Physics. [DOI: 10.1063/1.2798248]
\end{abstract}

The development of alternative high- $k$ gate dielectrics for future complimentary metal-oxide-semiconductor (CMOS) devices is indispensable for achieving both low leakage current and small equivalent oxide thickness. ${ }^{1}$ Recently, Hf oxide and Hf silicate have been receiving more and more attention for the forthcoming CMOS technologies. ${ }^{2-4}$ However, the $k$ values of Hf oxide and silicate are not high enough to satisfy the requirements of high$k$ gate dielectric. So, titanium was added into Hf oxide and silicate to increase the $k$ value because both Ti oxide and silicate have higher dielectric constant. ${ }^{5}$ As a result, HfTiON is a promising high $-k$ gate dielectric for small MOS devices. Besides small band offset with $\mathrm{Si}$, the small bandgap ${ }^{6,7}$ and poor thermodynamic stability ${ }^{8,9}$ of titanium oxide compared to hafnium oxide suggest that a buffer layer should be used to isolate the Ti atoms in HfTiON from the Si substrate. It is well known that $\mathrm{N}$-incorporated $\mathrm{Hf}$ silicate can suppress phase separation and microcrystallization, thus increasing the $k$ value and decreasing the leakage current. ${ }^{10}$ In this work, a stack structure of HfTiON on HfSiON is designed and fabricated as the high- $k$ gate dielectric of MOS device. Experimental results show that excellent electrical properties and reliability can be obtained for the stack gate dielectric structure.

MOS capacitor with HfTiON/HfSiON stack gate dielectric (denoted as the stack sample) was fabricated on (100)oriented $n$-type Si wafers with a doping concentration of 2 $\times 10^{17} \mathrm{~cm}^{-3}$. After RCA cleaning, the wafers were put in diluted $\mathrm{HF}$ for $1 \mathrm{~min}$ to remove the native $\mathrm{SiO}_{2}$. Then, the wafers were immediately loaded into the vacuum chamber of

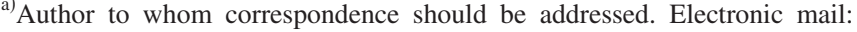
laip@eee.hku.hk a sputtering system made by DENTON Corporation. A HfN film $(\sim 2 \mathrm{~nm})$ was deposited at room temperature by the sputtering of a $\mathrm{Hf}$ target at $25 \mathrm{~W}$ rf power in an $\mathrm{Ar} / \mathrm{N}_{2}$ $(=24 / 6)$ ambient. Igniting pressure and sputtering pressure were 60 and 6.6 mTorr, respectively. Then, HfTiN was deposited at room temperature through cosputtering of $\mathrm{Hf}$ at $25 \mathrm{~W}$ rf power and Ti at $33 \mathrm{~W}$ dc power in the same ambient and pressure as for the deposition of HfN. Next, the sample was transferred to a postdeposition annealing (PDA) system. The PDA was performed in $\mathrm{N}_{2}(500 \mathrm{ml} / \mathrm{min})$ at $700{ }^{\circ} \mathrm{C}$ for $30 \mathrm{~s}$ to oxidize the HfN and HfTiN films by consuming the residual oxygen in the $\mathrm{N}_{2}$ ambient. During the PDA, Si out diffused into the buffer layer to form a HfSiON interlayer. A control sample with only HfTiON as gate dielectric (i.e., without the buffer layer, denoted as the control sample) was

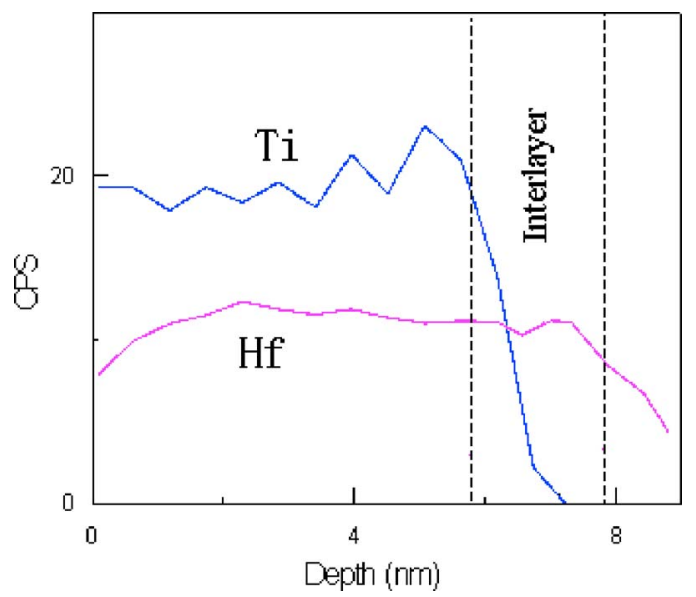

FIG. 1. XPS of the stack sample. 


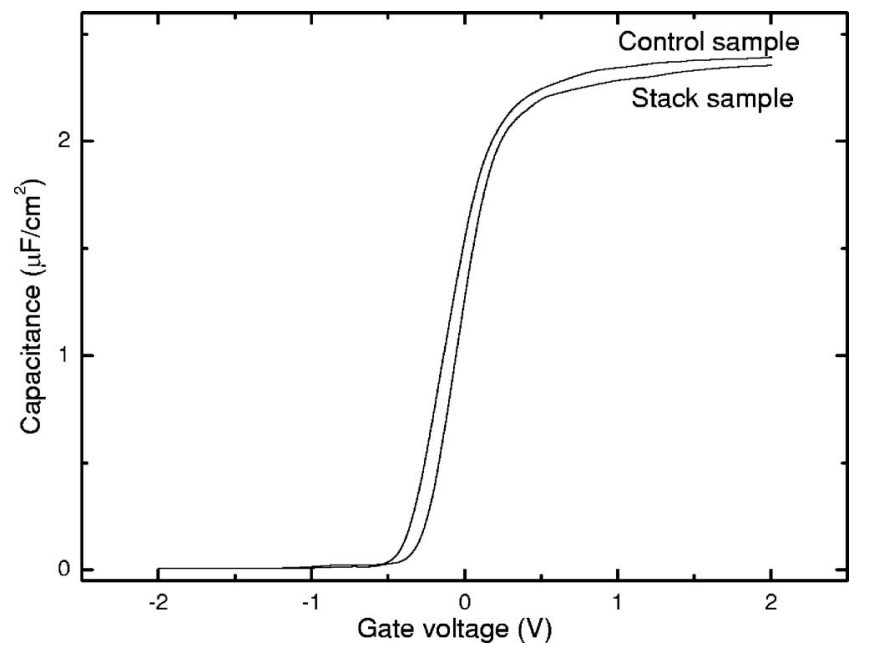

FIG. 2. Typical high-frequency $C-V$ curves of the two samples.

also fabricated. Then, $\mathrm{Al}$ was thermally evaporated and patterned using lithography technology as the gate electrode with an effective area of $7.85 \times 10^{-5} \mathrm{~cm}^{2}$. Also, Al was evaporated as the back electrode of the MOS devices to decrease contact resistance. Finally, both samples were annealed at $400{ }^{\circ} \mathrm{C}$ for $25 \mathrm{~min}$ in forming gas with $\mathrm{N}_{2} / \mathrm{H}_{2}$ $=95 / 5$.

High-frequency (1 MHz) $C$ - $V$ curves were measured at room temperature using HP4284A precision $L C R$ meter. Flatband voltage $\left(V_{\mathrm{fb}}\right)$, and oxide-charge density $\left(Q_{\mathrm{ox}}\right)$ were extracted from the high-frequency $C-V$ curves. The interfacestate density $\left(D_{\text {it }}\right)$ at midgap was also extracted from the high-frequency $C-V$ curve using the Terman method. ${ }^{11}$ Highfield stress $(2 \mathrm{MV} / \mathrm{cm}$ for $5000 \mathrm{~s})$, with the capacitors biased in accumulation by HP4156A precision semiconductor parameter analyzer, was used to examine device reliability under high-field stress in terms of flatband-voltage shift $\left(\Delta V_{\mathrm{fb}}\right)$ and gate-leakage increase. All measurements were carried out under a light-tight and electrically shielded condition.

The physical thickness $T_{\mathrm{ox}}$ of the high- $k$ gate dielectric is measured to be $7.8 \mathrm{~nm}$ by ellipsometry. Ti and Hf distribution profiles of the stack sample are analyzed by x-ray photoelectron spectroscopy (XPS), as shown in Fig. 1. Obviously, Ti sharply decreases near the HfTiON/HfSiON interface and cannot penetrate to the $\mathrm{Si}$ surface, resulting in little reaction between $\mathrm{Ti}$ and $\mathrm{Si}$. The $C$ - $V$ curves of the stack and control samples are shown in Fig. 2. Since the stack sample has an interlayer of HfSiON with a $k$ value lower than that of HfTiON, it exhibits slightly smaller accumulation capacitance compared to the control sample.

Electrical parameters of the two samples are extracted from the HF $C$ - $V$ curves and listed in Table I. The equivalent oxide-charge density $\left(Q_{\mathrm{ox}}\right)$, including fixed charges, bordertrap charges, mobile-ion charges, and interface-state charges, is calculated according to $Q_{\mathrm{ox}}=-C_{\mathrm{ox}}\left(V_{\mathrm{fb}}-\phi_{\mathrm{ms}}\right) / q$, where $\phi_{\mathrm{ms}}$ is the work-function difference between aluminum and

TABLE I. Electrical properties of the samples.

\begin{tabular}{lccccc}
\hline \hline Sample & $D_{\mathrm{it}}\left(10^{11} \mathrm{~cm}^{-2} \mathrm{eV}^{-1}\right)$ & $V_{\mathrm{fb}}(\mathrm{V})$ & $Q_{\mathrm{ox}}\left(10^{11} \mathrm{~cm}^{-2}\right)$ & $k$ & $\mathrm{CET}(\mathrm{nm})$ \\
\hline Stack & 2.15 & -0.11 & 5.69 & 19.7 & 1.51 \\
Control & 8.27 & -0.20 & 18.4 & 20.8 & 1.40 \\
\hline
\end{tabular}

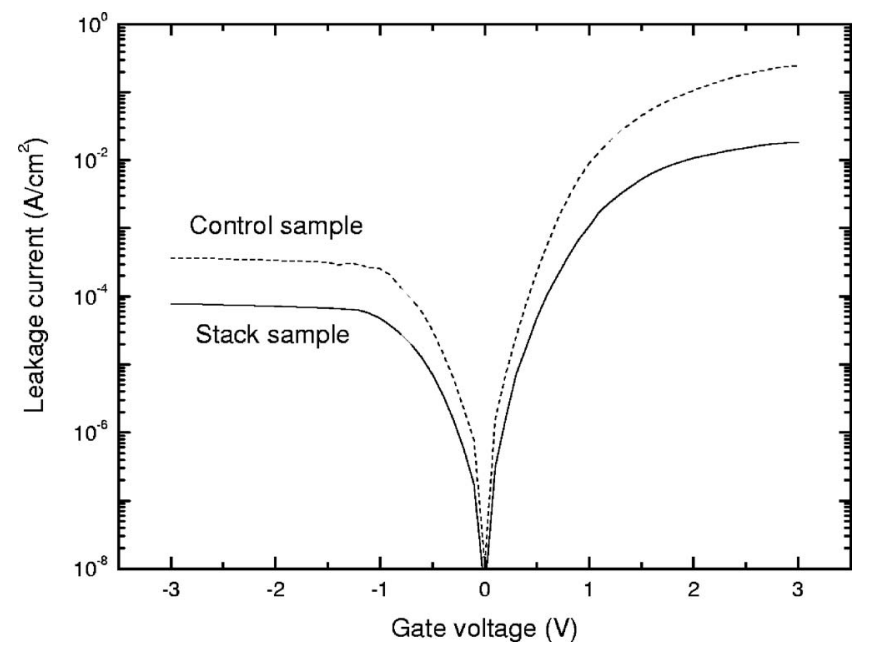

FIG. 3. Gate leakage current density of the two samples.

Si substrate; $C_{\mathrm{ox}}$ is oxide capacitance per unit area. For the control sample, large $Q_{\mathrm{ox}}$ implies that a large amount of defects are generated near the interface, probably due to the reaction of $\mathrm{Ti}$ with $\mathrm{Si}$ at the interface. ${ }^{9}$ For the stack sample, the reaction is greatly suppressed due to the isolation role of the HfSiON interlayer $(\sim 2 \mathrm{~nm})$, as shown in Fig. 1, resulting in smaller $Q_{\mathrm{ox}}$. Accordingly, a smaller $V_{\mathrm{fb}}$ shift is observed for the stack sample $(-0.11 \mathrm{~V})$ than the control sample $(-0.20 \mathrm{~V})$. Capacitance equivalent thickness (CET) can be obtained from the $C-V$ curve based on CET $=k_{0} k_{\mathrm{SiO}_{2}} / C_{\mathrm{ox}}$, where $k_{0}$ and $k_{\mathrm{SiO}_{2}}$ are dielectric constants of vacuum and $\mathrm{SiO}_{2}$, respectively. As compared to the control sample, smaller accumulation capacitance of the stack sample means a slightly larger CET. Moreover, the suppressed reaction between $\mathrm{Ti}$ and $\mathrm{Si}$ means that the stack sample has a lower interface-state density $\left(2.15 \times 10^{11}\right.$ $\left.\mathrm{cm}^{-2} \mathrm{eV}^{-1}\right)$ than the control sample $\left(8.27 \times 10^{11} \mathrm{~cm}^{-2} \mathrm{eV}^{-1}\right)$, due to the formation of a $\mathrm{SiO}_{2} / \mathrm{Si}$-like interface (i.e., $\mathrm{HfSiON} / \mathrm{Si}$ interface). The $k$ value of the stack sample is only slightly lower than that of the control sample due to the existence of the HfSiON interlayer.

Gate leakage current is another important device parameter. To evaluate the gate leakage performance of the devices, the $I-V$ curve is measured in both accumulation and inversion, as shown in Fig. 3. Oxide charges and especially inter-

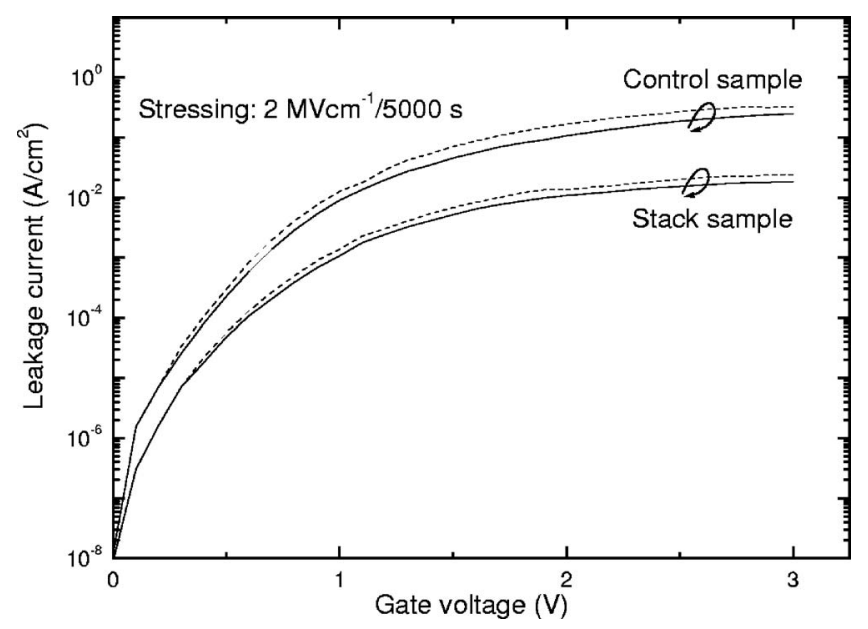

FIG. 4. Increase of gate leakage current after high-field stressing. 


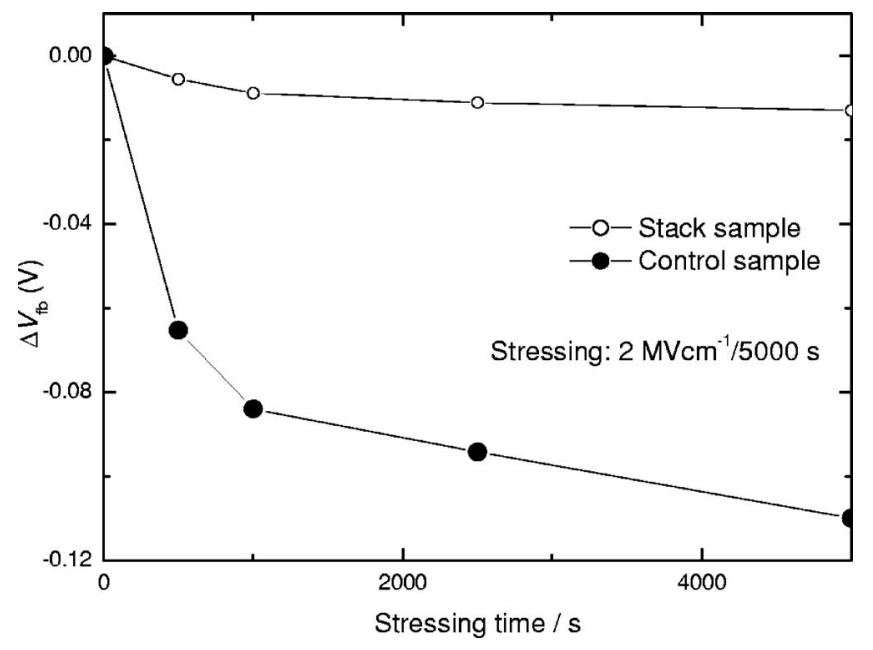

FIG. 5. Shift of flatband voltage $\left(\Delta V_{\mathrm{fb}}\right)$ during high-field stressing.

face states could be responsible for the leakage properties. Owing to its smaller $Q_{\mathrm{ox}}$ and $D_{\mathrm{it}}$, the stack sample exhibits lower gate leakage current than the control sample.

A high-field stressing at $2 \mathrm{MV} / \mathrm{cm}\left[=\left(V_{g}-V_{\mathrm{fb}}\right) / T_{\mathrm{ox}}\right.$, where $V_{g}$ is gate voltage] for $5000 \mathrm{~s}$ is used to evaluate the reliability of the devices. After stressing, increase of gate leakage current and shift of flatband voltage for the two samples are shown in Figs. 4 and 5, respectively. Obviously, the stack sample has much better reliability than the control sample due to the formation of a hardened oxynitrided interface without titanium silicide.

In summary, MOS device with HfTiON/HfSiON stack gate dielectric is fabricated, and excellent electrical properties are obtained, with lower interface-state/oxide-charge densities, lower gate leakage current, and better high-field reliability, as compared to the control sample without the HfSiON buffer. This is attributed to the HfSiON interlayer which effectively prevents $\mathrm{Ti}$ from penetrating to the Si surface, and thus forms an oxynitrided and $\mathrm{SiO}_{2} / \mathrm{Si}$-like interface. Therefore, the HfTiON/HfSiON stack dielectric is a promising technology for preparing high- $k$ gate-dielectric MOS devices with excellent electrical performances.

This work is financially supported by the National Natural Science Foundation of China (NSFC) under Grant No. 60376019, Open Foundation of State Key Laboratory of Advanced Technology for Materials Synthesis and Processing (Project No. WUT2006M02), and the University Development Fund (Nanotechnology Research Institute, 00600009) of the University of Hong Kong.

${ }^{1}$ G. D. Wilk, R. M. Wallace, and J. M. Anthony, J. Appl. Phys. 89, 5243 (2001).

${ }^{2}$ Y. Kim, G. Gebara, M. Freiler, and J. Barnett, Tech. Dig. - Int. Electron Devices Meet. 2001, 455.

${ }^{3}$ S. J. Rhee, C. S. Kang, and C. H. Choi, Tech. Dig. - Int. Electron Devices Meet. 2004, 837.

${ }^{4}$ K. Honda, A. Skaki, and M. Sakashita, Jpn. J. Appl. Phys., Part 1 43, 1571 (2004).

${ }^{5}$ J. Mcpherson, J. Kim, A. Shanware, H. Mogual, and J. Rodriguez, Tech. Dig. - Int. Electron Devices Meet. 2002, 633.

${ }^{6}$ G. Lueovsk, Microelectron. Reliab. 43, 1417 (2003).

${ }^{7}$ H. Huff, A. Hou, C. Lim, Y. Kim, J. Barnett, G. Bersuker, G. A. Brown, C. D. Young, P. M. Zeitzoff, J. Gutt, P. Lysaght, M. I. Gardner, and R. W. Murto, Microelectron. Eng. 69, 152 (2003).

${ }^{8}$ A. Shanware, M. R. Visokay, and J. J. Chambers, Tech. Dig. - Int. Electron Devices Meet. 2001, 137.

${ }^{9}$ M. Li, Z. Zhang, S. A. Campbell, W. L. Gladfelter, M. P. Agustin, D. O. Klenov, and S. Stemmer, J. Appl. Phys. 98, 054506 (2005).

${ }^{10}$ M. Yamaguchi, T. Sakoda, H. Minakata, S. Xiao, Y. Morisaki, K. Ikeda, and Y. Mishima, IEEE Trans. Electron Devices 53, 923 (2006).

${ }^{11}$ L. M. Terman, Solid-State Electron. 5, 285 (1962). 